\title{
Iterated Commutators for Multilinear Singular Integral Operators on Morrey Space with Non-Doubling Measures
}

\author{
Tie Li, Yinsheng Jiang, Yaoming Niu \\ Baotou Teacher's College of Inner Mongolia University of Science and Technology, Baotou, China \\ Email:690635457@qq.com
}

How to cite this paper: Li, T., Jiang, Y.S. and Niu, Y.M. (2020) Iterated Commutators for Multilinear Singular Integral Operators on Morrey Space with Non-Doubling Measures. Journal of Applied Mathematics and Physics, 8, 53-69.

https://doi.org/10.4236/jamp.2020.81005

Received: November 26, 2019

Accepted: December 28, 2019

Published: December 31, 2019

Copyright $\odot 2020$ by author(s) and Scientific Research Publishing Inc. This work is licensed under the Creative Commons Attribution International License (CC BY 4.0).

http://creativecommons.org/licenses/by/4.0/

\section{Abstract}

Let $\mu$ be a non-negative Radon measure on $\mathbb{R}^{d}$ which only satisfies the following growth condition that there exists a positive constant $C$ such that $\mu(B(x, r)) \leq C r^{n}$ for all $x \in \mathbb{R}^{d}, r>0$ and some fixed $n \in(0, d]$. This paper is interested in the properties of the iterated commutators of multilinear singular integral operators on Morrey spaces $M_{q}^{p}(\mu)$. Precisely speaking, we show that the iterated commutators generated by multilinear singular integrals operators $\left(T_{m}\right)_{\Pi \vec{b}}$ are bounded from $M_{q_{1}}^{p_{1}}(\mu) \times \cdots \times M_{q_{m}}^{p_{m}}(\mu)$ to $M_{q}^{p}(\mu)$ where $\vec{b} \in R B M O^{m}$ (Regular Bounded Mean Oscillation space) and $1<q_{j} \leq p_{j}<\infty$ with $1 / p=1 / p_{1}+\cdots+1 / p_{m}$ and $1 / q=1 / q_{1}+\cdots+1 / q_{m}$.

\section{Keywords}

Non-Doubling Measures, Morrey Space, Multilinear Singular Integral Operators, RBMO, Commutator

\section{Introduction}

Let $\mu$ be a positive Radon measures on $\mathbb{R}^{d}$ satisfying only the growth condition, that is, there exists a constant $C>0$ and $n \in(0, d]$ such that

$$
\mu(Q) \leq C l(Q)^{n}
$$

for any cube $Q \subset \mathbb{R}^{d}$ with sides parallel to the coordinate axes. $Q(x, l(Q))$ will be the cube centered at $x$ with side length $l(Q)$. For $r>0, r Q$ will denote the cube with the same center as $Q$ and with $l(r Q)=r l(Q)$. The set of all cubes $Q \subset \mathbb{R}^{d}$, satisfying $\mu(Q)>0$ is denoted by $\mathfrak{L}(\mu)$. In this note, we do not as- 
sume that $\mu$ is doubling.

Nazarov, Treil and Volberg developed the theory of the singular integrals for the measures with growth condition to investigate the analytic capacity on the complex plane [1] [2]. Tolsa showed that the analytic capacity is subadditive and that it is bi-Lipschitz invariant [3] [4] and defined for the growth measures $R B M O$ (regular bounded mean oscillation) space, the Hardy space $H_{1}(\mu)$ and the Littlewood-Paley decomposition [5] [6]. He also gave his $H_{1}(\mu)$ space in terms of the grand maximal operator [7]. Recently many people paid attention to the measure with growth condition because of recovering the CalderónZygmund theory and solving the long-standing open Painlevé problem.

The boundedness of fractional integral operators on the classical Morrey spaces was studied by Adams [8], Chiarenza et al. [9]. In [9], by establishing a pointwise estimate of fractional integrals in terms of the Hardy-Littlewood maximal function, they showed the boundedness of fractional integral operators on the Morrey spaces. In 2005, Sawano and Tanaka [10] gave a natural definition of Morrey spaces for Radon measures which might be non-doubling but satisfied the growth condition, and they investigated the boundedness in these spaces of some classical operators in harmonic analysis. Later on, Sawano [11] defined the generalized Morrey spaces on $\mathbb{R}^{n}$ for non-doubling measure and showed the properties of maximal operators, fractional integral operators and singular operators in this space.

A classical result of commutator is due to Coifman, Rochberg and Weiss [12], if $b \in B M O$ and $T$ is a Calderón-Zygmund operator, then the commutator $[b, T]$ is bounded on $L^{p}$ spaces for $1<p<\infty$. The same result for the multilinear commutator was obtained by Pérez and Trujillo-Gonzalez [13]. Tolsa [5] developed the theory of Calderón-Zygmund operators and their commutators with $R B M O$ functions in the setting of non-doubling measures. Hu, Meng and Yang [14] considered the multilinear commutator on Lebesgue spaces with non-doubling measures. Chen and Sawyer [15] modified the definition of $R B M O$ to investigate the commutators of the potential operators and $R B M O$ functions.

In the last decade, multilinear singular integrals of Calderón-Zygmund type have attracted great attentions. Some interesting results refer to [16] [17] [18] [19] [20] in the text of Lebesgue measures. It points out that Perez and Pradolini [21] introduced a said iterated commutators generated by the multilinear singular integral operators with Calderón-Zygmund type and vector function $\vec{b} \in R B M O^{m}$ and obtained the boundedness from $L^{p_{1}} \times \cdots \times L^{p_{m}}$ to $L^{p}$ with $1 / p=1 / p_{1}+\cdots+1 / p_{m}$ for $1<p_{1}, \cdots, p_{m}<\infty$ (in fact, they considered the weighted case). $\mathrm{Xu}$ [22] extended the result to the case of the non-doubling measures. Very recently, Tao and $\mathrm{He}$ [23] obtained the boundedness of the multilinear Calderón-Zygmund operators on the generalized Morrey spaces over the quasi-metric space of non-homogeneoustype. The aim of this paper is to study the iterated commutators of multilinear singular integral operators on Morrey 
spaces with non-doubling measures.

Before stating our result, we recall some definitions and notation. Given $\beta_{d} \geq 2^{d+1}$ large enough but depending only on the dimension $d$, we say that a cube $Q \subset \mathbb{R}^{d}$ is doubling if $\mu(2 Q) \leq \beta_{d} \mu(Q)$. For any fixed cube $Q \subset \mathbb{R}^{d}$, let $N$ be the smallest nonnegative integer such that $2^{N} Q$ is doubling. We denote this cube by $\tilde{Q}$.

For two cubes $Q \subset R$ in $\mathbb{R}^{d}$, we suppose

$$
K_{Q, R}=1+\sum_{k=1}^{N_{Q, R}} \frac{\mu\left(2^{k} Q\right)}{l\left(2^{k} Q\right)^{n}}
$$

where $N_{Q, R}$ is the first positive integer $k$ such that $l\left(2^{k} Q\right) \geq l(R)$. This was introduced by Tolsa in [5].

Let $m_{Q} f$ be the mean value of $f$ on $Q$, namely, $m_{Q} f=\frac{1}{\mu(Q)} \int_{Q} f(x) \mathrm{d} \mu$. The regularity bounded mean oscillations function spaces were introduced by Tolsa [5].

Definition 1.1. Let $\eta>1$ be a fixed constant. We say that $f \in L_{l o c}^{1}(\mu)$ is in $R B M O$ if there exists a constant $\mathcal{A}$ such that

$$
\frac{1}{\mu(\eta Q)} \int_{Q}\left|f(y)-m_{\tilde{Q}} f\right| \mathrm{d} \mu(y) \leq \mathcal{A},
$$

for any cube $Q$, and

$$
\left|m_{Q} f-m_{R} f\right| \leq \mathcal{A} K_{Q, R},
$$

for any two doubling cubes $Q \subset R$. The mininal constant $\mathcal{A}$ is the $\operatorname{RBMO}(\mu)$ norm of $f$, and it will be denoted by $\|f\|_{*}$.

The definition of the Morrey space with non-doubling measure is given in the following [10].

Definition 1.2. Let $k>1$ and $1 \leq q \leq p<\infty$, the Morrey space $M_{q}^{p}(k, \mu)$ is defined as

$$
M_{q}^{p}(k, \mu):=\left\{f \in L_{l o c}^{q}(\mu):\|f\|_{M_{q}^{p}(k, \mu)}<\infty\right\}
$$

where

$$
\|f\|_{M_{q}^{p}(k, \mu)}:=\sup _{Q \in \mathfrak{L}(\mu)} \mu(k Q)^{\frac{1}{p}-\frac{1}{q}}\left(\int_{Q}|f|^{q} \mathrm{~d} \mu\right)^{\frac{1}{q}} .
$$

It is easy to observe that $L^{p}(\mu)=M_{p}^{p}(k, \mu)$, and Hölder's inequality tells us $\|f\|_{M_{q_{2}}^{p}(k, \mu)} \leq\|f\|_{M_{q_{1}}^{p}(k, \mu)}$ for all $1 \leq q_{2} \leq q_{1} \leq p$, then we have $L^{p}(\mu)=M_{p}^{p}(k, \mu) \subset M_{q_{1}}^{p}(k, \mu) \subset M_{q_{2}}^{p}(k, \mu)$. The space $M_{q}^{p}(k, \mu)$ is a Banach space with its norm $\|f\|_{M_{q}^{p}(k, \mu)}$ and the parameter $k>1$ appearing in the definition does not affect it. The Morrey space norm reflects local regularity of $f$ more precisely than the Lebesgue space norm. See [10] [11] [24] for details. We will denote $M_{q}^{p}(2, \mu)$ by $M_{q}^{p}(\mu)$. 
Denoting by $\vec{f}=\left(f_{1}, f_{2}, \cdots, f_{m}\right)$, we consider the multilinear singular integral operator $T_{m}$ as follows,

$$
\begin{aligned}
& T_{m}(\vec{f})(x) \\
& =\int_{\left(\mathbb{R}^{d}\right)^{m}} K\left(x, y_{1}, \cdots, y_{m}\right) f_{1}\left(y_{1}\right) \cdots f_{m}\left(y_{m}\right) \mathrm{d} \mu\left(y_{1}\right) \cdots \mathrm{d} \mu\left(y_{m}\right) \text { for } x \in \mathbb{R}^{d},
\end{aligned}
$$

whenever $f_{1}, \cdots, f_{m}$ are $C^{\infty}$-functions with compact support and $x \notin \bigcap_{j=1}^{m} \operatorname{supp} f_{j}$. Moreover,

$$
\left|K\left(y_{0}, y_{1}, \cdots, y_{m}\right)\right| \leq A\left(\sum_{k, l=0}^{m}\left|y_{k}-y_{l}\right|\right)^{-m n}
$$

and, for some $\epsilon>0$,

$$
\left|K\left(y_{0}, y_{1}, \cdots, y_{j}, \cdots, y_{m}\right)-K\left(y_{0}, y_{1}, \cdots, y_{j}^{\prime}, \cdots, y_{m}\right)\right| \leq \frac{A\left|y_{j}-y_{j}^{\prime}\right|^{\epsilon}}{\left(\sum_{k, l=0}^{m}\left|y_{k}-y_{l}\right|\right)^{m n+\epsilon}}
$$

provided that $0 \leq j \leq m$ and $\left|y_{j}-y_{j}^{\prime}\right| \leq \frac{1}{2} \max _{0 \leq k \leq m}\left|y_{j}-y_{k}\right|$.

Let $b_{i} \in R B M O(\mu)$ for $i=1,2, \cdots, m$ and let $\vec{b}=\left(b_{1}, b_{2}, \cdots, b_{m}\right)$, then the iterated commutator $\left(T_{m}\right)_{\Pi \vec{b}}$ is formally defined as

$$
\begin{aligned}
\left(T_{m}\right)_{\prod^{\vec{b}}}(\vec{f})(x)= & \int_{\left(\mathbb{R}^{d}\right)^{m}} \prod_{j=1}^{m}\left(b_{j}(x)-b_{j}\left(y_{j}\right)\right) K\left(x, y_{1}, \cdots, y_{m}\right) \\
& \times f_{1}\left(y_{1}\right) \cdots f_{m}\left(y_{m}\right) \mathrm{d} \mu\left(y_{1}\right) \cdots \mathrm{d} \mu\left(y_{m}\right) .
\end{aligned}
$$

Suppose $\|\mu\|=\infty$ the main result in this paper can be stated as follow.

Theorem 1.1. Let $\left(T_{m}\right)_{\prod_{\vec{b}}}$ as in (9) and satisfying conditions (7) and (8). Let $1<q_{j} \leq p_{j}<\infty$ with $\frac{1}{p}=\frac{1}{p_{1}}+\cdots+\frac{1}{p_{m}}$ and $\frac{1}{q}=\frac{1}{q_{1}}+\cdots+\frac{1}{q_{m}}$. Suppose $b_{i} \in \operatorname{RBMO}(\mu)$ for $i=1,2, \cdots, m$. If $T_{m}$ maps $L^{1}(\mu) \times \cdots \times L^{1}(\mu)$ to $L^{1 / m, \infty}(\mu)$, then the commutators $\left(T_{m}\right)_{\Pi_{\vec{b}}}$ are bounded from $M_{q_{1}}^{p_{1}}(\mu) \times \cdots \times M_{q_{m}}^{p_{m}}(\mu)$ to $M_{q}^{p}(\mu)$, that is,

$$
\left\|\left(T_{m}\right)_{\prod_{\vec{b}}}(\vec{f})(x)\right\|_{M_{q}^{p}(\mu)} \leq C \prod_{i=1}^{m}\left\|b_{i}\right\|_{*}\left\|f_{i}\right\|_{M_{q_{i}}^{p_{i}}(\mu)} .
$$

More generally, denote by $C_{i}^{m}$ the family of all subsets $\sigma=\left\{\sigma_{1}, \sigma_{2}, \cdots, \sigma_{i}\right\}$ of $i$ different elements of $\{1,2, \cdots, m\}$, and let $\sigma^{\prime}=\{1,2, \cdots, m\} \backslash \sigma$ and $\vec{b}_{\sigma}=\left\{b_{\sigma_{1}}, b_{\sigma_{2}}, \cdots, b_{\sigma_{i}}\right\}$. For any $\sigma \in C_{i}^{m}$, we define

$$
\begin{aligned}
\left(T_{m}\right)_{\Pi \vec{b}_{\sigma}}(\vec{f})(x)= & \int_{\left(\mathbb{R}^{d}\right)^{m}} \prod_{\sigma_{j} \in \sigma}\left(b_{\sigma_{j}}(x)-b_{\sigma_{j}}\left(y_{\sigma_{j}}\right)\right) K\left(x, y_{1}, \cdots, y_{m}\right) \\
& \times f_{1}\left(y_{1}\right) \cdots f_{m}\left(y_{m}\right) \mathrm{d} \mu\left(y_{1}\right) \cdots \mathrm{d} \mu\left(y_{m}\right) .
\end{aligned}
$$

In case $\sigma=\{1,2, \cdots, m\}$, one sees that $\left(T_{m}\right)_{\Pi \vec{b}_{\sigma}}$ is just the commutator $\left(T_{m}\right)_{\Pi_{b} b}$. So we have a more generalization version of the theorem as following.

Theorem 1.2. Let $\left(T_{m}\right)_{\Pi \vec{b}_{\sigma}}$ as in (11) and satisfying conditions (7) and (8). Let $1<q_{j} \leq p_{j}<\infty$ with $\frac{1}{p}=\frac{1}{p_{1}}+\cdots+\frac{1}{p_{m}}$ and $\frac{1}{q}=\frac{1}{q_{1}}+\cdots+\frac{1}{q_{m}}$. Suppose 
$b_{i} \in \operatorname{RBMO}(\mu)$ for $i=1,2, \cdots, m$. If $T_{m}$ maps $L^{1}(\mu) \times \cdots \times L^{1}(\mu)$ to

$L^{1 / m, \infty}(\mu)$, then for all $\sigma \subset\{1,2, \cdots, m\}$, the commutators $\left(T_{m}\right)_{\Pi \vec{b}_{\sigma}}$ are bounded from $M_{q_{1}}^{p_{1}}(\mu) \times \cdots \times M_{q_{m}}^{p_{m}}(\mu)$ to $M_{q}^{p}(\mu)$, that is,

$$
\left\|\left(T_{m}\right)_{\prod_{b_{\sigma}}}(\vec{f})(x)\right\|_{M_{q}^{p}(\mu)} \leq C \prod_{j \in \sigma}\left\|b_{j}\right\|_{*} \prod_{i=1}^{m}\left\|f_{i}\right\|_{M_{q_{i}}^{p_{i}}(\mu)} .
$$

\section{Proof of Main Results}

Before proving our theorem, we recall the following maximal operator,

$$
M_{k} f(x)=\sup _{x \in Q \subset \mathfrak{L}(\mu)} \frac{1}{\mu(k Q)} \int_{Q}|f(y)| \mathrm{d} \mu(y) .
$$

we will use the sharp maximal estimates. Let $f$ be a function in $L_{l o c}^{1}(\mu)$, the sharp maximal function of $f$ is defined by

$$
M^{\#} f(x)=\sup _{Q \ni x} \frac{1}{\mu\left(\frac{3}{2} Q\right)} \int_{Q}\left|f(y)-m_{\tilde{Q}} f\right| \mathrm{d} \mu(y)+\sup _{\substack{R>Q \ni x \\ Q, R \text { doubling }}} \frac{\left|m_{Q} f-m_{R} f\right|}{K_{Q, R}}
$$

The non-centered doubling maximal operator is defined by

$$
N f(x)=\sup _{Q \ni x, Q \text { doubling }} \frac{1}{\mu(Q)} \int_{Q}|f(y)| \mathrm{d} \mu(y) .
$$

By the Lebesgue differential theorem, it is easy to see that $|f(x)| \leq N f(x)$ for any $f \in L_{l o c}^{1}(\mu)$ and $\mu$-a.e.x $\in \mathbb{R}^{d}$. Define the non-centered maximal operator,

$$
M_{\tau, \eta} f(x)=\sup _{Q \ni x}\left\{\frac{1}{\mu(\eta Q)} \int_{Q}|f(y)|^{\tau} \mathrm{d} \mu(y)\right\}^{\frac{1}{\tau}} .
$$

for $\eta>1$ and $\tau>1$, where the supremum is taking over all the cubes $Q$ containing the point $x$.

To prove Theorem 1.2 is reduced to the following lemmas.

Lemma 2.1. Let $\tau>1, s_{i}>1, b_{i} \in R B M O(\mu)$ and $f_{i} \in L^{q_{i}}(\mu), i=1,2, \cdots, m$ and $\sigma \subset\{1,2, \cdots, m\}$. If $T_{m}$ maps $L^{1}(\mu) \times \cdots \times L^{1}(\mu)$ to $L^{1 / m, \infty}(\mu)$ and satisfying conditions (7) and (8). Then we have

$$
\begin{aligned}
M^{\#} & \left(\left(T_{m}\right)_{\prod \vec{b}_{\sigma}}(\vec{f})\right)(x) \\
\leq & C\left[\prod_{j \in \sigma}\left\|b_{j}\right\|_{*} M_{\tau, \frac{3}{2}}\left(T_{m}(\vec{f})\right)(x)\right. \\
& +\sum_{\substack{\sigma_{1} \cup \sigma_{2}=\sigma \\
\sigma_{1} \neq \varnothing, \sigma_{2} \neq \varnothing}} \prod_{j \notin \sigma_{1}}\left\|b_{j}\right\|_{*} M_{\tau, \frac{3}{2}}\left(T_{m}\right)_{\prod \vec{b}_{\sigma_{2}}}(\vec{f})(x) \\
& \left.+\prod_{j \in \sigma}\left\|b_{j}\right\|_{*} \prod_{j=1}^{m} M_{s_{j}, \frac{9}{8}} f_{j}(x)\right] .
\end{aligned}
$$

We postpone the proof of Lemma 2.1 after of Theorem 1.2. 
Lemma 2.2. [10] Let $q>\tau>1, \eta>1$, and $1<q \leq p<\infty$, then the operator $M_{\tau, \eta}$ is bounded on $M_{q}^{p}(\mu)$ and

$$
\left\|M_{\tau, \eta}(f)\right\|_{M_{q}^{p}(\mu)} \leq C\|f\|_{M_{q}^{p}(\mu)} .
$$

with the constant $C$ independent of $f$.

Lemma 2.3. [24] Suppose that $1<q \leq p<\infty$, and there exists an increasing sequence of concentric doubling cubes, $I_{0} \subset I_{1} \subset \cdots \subset I_{k} \subset \cdots$, such that

$$
\lim _{k \rightarrow \infty} m_{I_{k}}(f)=0 \text { and } \bigcup_{k=0}^{\infty} I_{k}=\mathbb{R}^{d}
$$

Then there exist a constant $C>0$ independent on $f$ such that

$$
\|N f\|_{M_{q}^{p}(\mu)} \leq C\left\|M^{\#}\right\|_{M_{q}^{p}(\mu)} .
$$

Lemma 2.4. [25] Let $1<p_{i}<\infty$ and $\frac{1}{p}=\frac{1}{p_{1}}+\cdots+\frac{1}{p_{m}}$. If $T_{m}$ maps $L^{1}(\mu) \times \cdots \times L^{1}(\mu)$ to $L^{1 / m, \infty}(\mu)$ and satisfying conditions (7) and (8). Then there exists a constant $C$ independent of $f_{i}$ such that

$$
\left\|T_{m}(\vec{f})\right\|_{L^{p}(\mu)} \leq C \prod_{i=1}^{m}\left\|f_{i}\right\|_{L^{p_{i}}(\mu)} .
$$

Remark 2.1. If $T_{m}$ maps $L^{1}(\mu) \times \cdots \times L^{1}(\mu)$ to $L^{1 / m, \infty}(\mu)$ and satisfying conditions (7) and (8), with $1<q_{i} \leq p_{i}<\infty, \frac{1}{p}=\frac{1}{p_{1}}+\cdots+\frac{1}{p_{m}}$ and $\frac{1}{q}=\frac{1}{q_{1}}+\cdots+\frac{1}{q_{m}}$. From Corollary 1.8 in [23], we can easily get that $T_{m}$ is bounded from $M_{q_{1}}^{p_{1}}(\mu) \times \cdots \times M_{q_{m}}^{p_{m}}(\mu)$ to $M_{q}^{p}(\mu)$.

Proof of Theorem 1.2. Using Lemma 2.1, Lemma 2.2, Lemma 2.3 and Lemma 2.4 , we get that

$$
\begin{aligned}
& \left\|\left(T_{m}\right)_{b_{i}}(\vec{f})\right\|_{M_{q}^{p}(\mu)} \\
& \leq C\left\|N\left(\left(T_{m}\right)_{b_{i}}(\vec{f})\right)\right\|_{M_{q}^{p}(\mu)} \leq C\left\|M^{\#}\left(\left(T_{m}\right)_{b_{i}}(\vec{f})\right)\right\|_{M_{q}^{p}(\mu)} \\
& \leq C\left\|b_{i}\right\|_{*}\left\|M_{\tau, \frac{3}{2}}\left(T_{m}(\vec{f})\right)(x)+\prod_{i=1}^{m} M_{s_{i}, \frac{9}{8}} f_{i}\right\|_{M_{q}^{p}(\mu)} \\
& \leq C\left\|b_{i}\right\|_{*}\left\|M_{\tau, \frac{3}{2}}\left(T_{m}(\vec{f})\right)\right\|_{M_{q}^{p}(\mu)}+C\left\|b_{i}\right\|_{*}\left\|\prod_{i=1}^{m} M_{s_{i}, \frac{9}{8}} f_{i}\right\|_{M_{q}^{p}(\mu)} \\
& \leq C\left\|b_{i}\right\|_{*}\left\|T_{m}(\vec{f})\right\|_{M_{q}^{p}(\mu)}+C\left\|b_{i}\right\|_{*} \prod_{i=1}^{m}\left\|f_{i}\right\|_{M_{q_{j}}^{p_{j}}(\mu)} \\
& \leq C\left\|b_{i}\right\|_{*} \prod_{j=1}^{m}\left\|f_{i}\right\|_{M_{q_{j}}^{p_{j}}(\mu)} .
\end{aligned}
$$

Applying the inequality (17) in Lemma 2.1, for $\sigma \subset\{1,2, \cdots, m\}$, we have 


$$
\begin{aligned}
& \left\|\left(T_{m}\right)_{\prod_{\vec{b}}}(\vec{f})\right\|_{M_{q}^{p}(\mu)} \\
& \leq C\left\|N\left(\left(T_{m}\right)_{\prod_{b}}(\vec{f})\right)\right\|_{M_{q}^{p}(\mu)} \leq C\left\|M^{\#}\left(\left(T_{m}\right)_{\prod_{\vec{b}}}(\vec{f})\right)\right\|_{M_{q}^{p}(\mu)} \\
& \leq C\left\|\prod_{j \in \sigma}\right\| b_{j}\left\|_{*} M_{\tau, \frac{3}{2}}\left(T_{m}(\vec{f})\right)+\sum_{\substack{\sigma_{1} \cup \sigma_{2}=\sigma \\
\sigma_{1} \neq \varnothing \sigma_{2} \neq \varnothing}} \prod_{j \in \sigma_{1}}\right\| b_{j} \|_{*} M_{\tau, \frac{3}{2}}\left(\left(T_{m}\right)_{\prod \vec{b}_{\sigma_{2}}}(\vec{f})\right) \\
& +\prod_{j \in \sigma}\left\|b_{j}\right\|_{*} \prod_{j=1}^{m} M_{s_{j}, \frac{9}{8}} f_{j} \|_{M_{q}^{p}(\mu)} \\
& \leq C \prod_{j \in \sigma}\left\|b_{j}\right\|_{*} \prod_{j=1}^{m}\left\|f_{j}\right\|_{M_{q_{j}}^{p_{j}}(\mu)}+C \sum_{\substack{\sigma_{1} \cup \sigma_{2}=\sigma \\
\sigma_{1} \neq \varnothing \sigma_{2} \neq \varnothing}} \prod_{j \in \sigma_{1}}\left\|b_{j}\right\|_{*}\left\|M_{\tau, \frac{3}{2}}\left(\left(T_{m}\right)_{\prod \vec{b}_{\sigma_{2}}}(\vec{f})\right)\right\|_{M_{q}^{p}(\mu)} \\
& \leq C \prod_{j \in \sigma}\left\|b_{j}\right\|_{*} \prod_{j=1}^{m}\left\|f_{j}\right\|_{M_{q_{j}}^{p_{j}}(\mu)}+C \sum_{\substack{\sigma_{1} \cup \sigma_{2}=\sigma \\
\sigma_{1} \neq \varnothing \sigma_{2} \neq \varnothing}} \prod_{j \in \sigma_{1}}\left\|b_{j}\right\|\left\|_{*}\right\|\left(T_{m}\right)_{\prod \vec{b}_{\sigma_{2}}}(\vec{f}) \|_{M_{q}^{p}(\mu)}
\end{aligned}
$$

where $\sigma_{1}$ and $\sigma_{2}$ are two nonempty subsets of $\sigma$ and $\sigma_{1} \cap \sigma_{2}=\varnothing$. Hence, we can make use of induction on $\sigma \subset\{1,2, \cdots, m\}$ to get that

$$
\left\|\left(T_{m}\right)_{\prod_{\vec{b}_{\sigma}}}(\vec{f})\right\|_{M_{q}^{p}(\mu)} \leq C \prod_{j \in \sigma}\left\|b_{j}\right\|_{*} \prod_{i=1}^{m}\left\|f_{i}\right\|_{M_{q_{i}}^{p_{i}}(\mu)} .
$$

This completes the proof of Theorem 1.2.

For simplicity of the notation, we only show the special case $\sigma=\{1, \cdots, m\}$ of Lemma 2.1. The similar process with minor modification will be to able prove Lemma 2.1 for the general case.

Lemma 2.5. Let $\tau>1, s_{i}>1, b_{i} \in R B M O(\mu)$ and $f_{i} \in L^{q_{i}}(\mu), i=1,2, \cdots, m$. If $T_{m}$ maps $L^{1}(\mu) \times \cdots \times L^{1}(\mu)$ to $L^{1 / m, \infty}(\mu)$ and satisfying conditions (7) and (8), then there exists a constant $C>0$ independent of $b_{i}$ and $f_{i}$ such that

$$
\begin{aligned}
M^{\#} & \left(\left(T_{m}\right)_{\prod^{b}}(\vec{f})\right)(x) \\
\leq & C\left[\prod_{i=1}^{m}\left\|b_{i}\right\|_{*} M_{\tau, \frac{3}{2}}\left(T_{m}(\vec{f})\right)(x)\right. \\
& +\sum_{i=1}^{m-1} \sum_{\sigma \in C_{i}^{m}} \prod_{j \in \sigma}\left\|b_{j}\right\|_{*} M_{\eta, \frac{3}{2}}\left(\left(T_{m}\right)_{\prod_{b_{\sigma^{\prime}}}}(\vec{f})\right)(x) \\
& \left.+\prod_{i=1}^{m}\left\|b_{i}\right\|_{*} M_{s_{i}, \frac{9}{8}} f_{i}(x)\right]
\end{aligned}
$$

In order to prove Lemma 2.5, we have the following decomposition for the commutators $\left(T_{m}\right)_{\Pi_{\vec{b}}}$. For any $\vec{\lambda}=\left(\lambda_{1}, \lambda_{2}, \cdots, \lambda_{m}\right) \in \mathbb{R}^{m}$, writing $\vec{b}_{\sigma}(x)-\vec{b}_{\sigma}(y)=\left(\vec{b}_{\sigma}(x)-\vec{\lambda}_{\sigma}\right)-\left(\vec{b}_{\sigma}(y)-\vec{\lambda}_{\sigma}\right)$, thus it is clear that $\left(T_{m}\right)_{\prod \vec{b}_{\sigma}}(\vec{f})=\left(T_{m}\right)_{\Pi\left(\vec{b}_{\sigma}-\vec{\lambda}_{\sigma}\right)}(\vec{f})$. Moreover,

$$
\begin{aligned}
& \left(T_{m}\right)_{\prod_{\vec{b}}}(\vec{f})(x) \\
& =\sum_{i=0}^{m} \sum_{\sigma \in C_{i}^{m}}(-1)^{m-i} \prod_{\sigma_{j} \in \sigma}\left(b_{\sigma_{j}}(x)-\lambda_{\sigma_{j}}\right) \times \int_{\left(\mathbb{R}^{d}\right)^{m}} \prod_{\sigma_{k} \in \sigma^{\prime}}\left(b_{\sigma_{k}}(y)-\lambda_{\sigma_{k}}\right) \\
& \quad \times K\left(x, y_{1}, \cdots, y_{m}\right) f_{1}\left(y_{1}\right) \cdots f_{m}\left(y_{m}\right) \mathrm{d} \mu\left(y_{1}\right) \cdots \mathrm{d} \mu\left(y_{m}\right) .
\end{aligned}
$$


By expanding $b_{\sigma_{k}}(y)-\lambda_{\sigma_{k}}=\left(b_{\sigma_{k}}(y)-b_{\sigma_{k}}(x)\right)-\left(b_{\sigma_{k}}(x)-\lambda_{\sigma_{k}}\right)$ and

$$
\begin{aligned}
& \prod_{\sigma_{k} \in \sigma^{\prime}}\left(b_{\sigma_{k}}(y)-\lambda_{\sigma_{k}}\right) \\
= & \sum_{\substack{\sigma^{(1)} \cup \sigma^{(2)}=\sigma^{\prime} \\
\sigma^{(1)} \cap \sigma^{(2)}=\varnothing}} \prod_{\sigma_{u} \in \sigma^{(1)}}\left(b_{\sigma_{u}}(y)-b_{\sigma_{u}}(x)\right) \prod_{\sigma_{v} \in \sigma^{(2)}}\left(b_{\sigma_{v}}(x)-\lambda_{\sigma_{v}}\right),
\end{aligned}
$$

hence we can obtain from the equality (20) that

$$
\begin{aligned}
& \left(T_{m}\right)_{\prod_{\vec{b}}}(\vec{f})(x) \\
& =\prod_{j=1}^{m}\left(b_{j}(x)-\lambda_{j}\right) T_{m}(\vec{f})(x)+(-1)^{m} T_{m}((\vec{b}-\vec{\lambda}) \vec{f})(x) \\
& \quad+\sum_{i=1}^{m-1} \sum_{\sigma \in C_{i}^{m}} C_{m, i} \prod_{\sigma_{j} \in \sigma}\left(b_{\sigma_{j}}(x)-\lambda_{\sigma_{j}}\right) \times\left(T_{m}\right)_{\prod \vec{b}_{\sigma^{\prime}}}(\vec{f})(x)
\end{aligned}
$$

where $C_{m, i}$ are constants depending only on $m$ and $i$.

Proof of Lemma 2.5. For simplicity, we denote by $\mathcal{R}(\vec{b}, \vec{f})(x)$ the quantities on the right hand side of the inequality (19). Recall the definition of the sharp maximal operator $M^{\#}$, and use the standard technique, see [15] for example, we only need to prove that

$$
\frac{1}{\mu\left(\frac{3}{2} Q\right)} \int_{Q}\left|\left(T_{m}\right)_{\Pi \vec{b}}(\vec{f})(z)-h_{Q}\right| \mathrm{d} \mu(z) \leq C \mathcal{R}(\vec{b}, \vec{f})(x)
$$

and

$$
\left|h_{Q}-h_{R}\right| \leq C\left(K_{Q, R}\right)^{2 m} \mathcal{R}(\vec{b}, \vec{f})(x)
$$

with the absolute constant $\mathrm{C}$ independent of $\vec{b}, \vec{f}, Q$ and $R$, where $R$ is any doubling cube with $Q \subset R$. In fact, we take

$$
h_{Q}=(-1)^{m} m_{Q}\left(T_{m}\left(\left(m_{\tilde{Q}}\left(b_{1}\right)-b_{1}\right) f_{1} \chi_{\mathbb{R}^{d}, \frac{4}{3} Q}, \cdots,\left(m_{\tilde{Q}}\left(b_{m}\right)-b_{m}\right) f_{m} \chi_{\mathbb{R}^{d} \backslash \frac{4}{3} Q}\right)\right)
$$

and clearly

$$
h_{R}=(-1)^{m} m_{R}\left(T_{m}\left(\left(m_{\tilde{R}}\left(b_{1}\right)-b_{1}\right) f_{1} \chi_{\mathbb{R}^{d} \backslash \frac{4}{3} R}, \cdots,\left(m_{\tilde{R}}\left(b_{m}\right)-b_{m}\right) f_{m} \chi_{\mathbb{R}^{d} \backslash \frac{4}{3} R}\right)\right)
$$

Recall the equality (21), for any $z \in Q$, we have that

$$
\begin{aligned}
& \left|\left(T_{m}\right)_{\prod_{\vec{b}}}(\vec{f})(z)-h_{Q}\right| \\
& \leq\left|\prod_{j=1}^{m}\left(b_{j}(x)-m_{\tilde{Q}}\left(b_{j}\right)\right) T_{m}(\vec{f})(z)\right| \\
& \quad+\left|\sum_{i=1}^{m-1} \sum_{\sigma \in C_{i}^{m}} C_{i, m} \prod_{\sigma_{j} \in \sigma}\left(b_{\sigma_{j}}(z)-m_{\tilde{Q}}\left(b_{\sigma_{j}}\right)\right)\left(T_{m}\right)_{\prod_{b_{\sigma^{\prime}}}}(\vec{f})(z)\right| \\
& \quad+\left|(-1)^{m} T_{m}\left(\left(\vec{b}-m_{\tilde{Q}}(\vec{b})\right) \vec{f}\right)(z)-h_{Q}\right| \\
& =: I(z)+I I(z)+I I I(z) .
\end{aligned}
$$


In order to show the inequality (22), we will calculate the integrals for the three functions above, respectively. Firstly, for $\tau>1$, by the Hölder inequality one sees that

$$
\begin{aligned}
& \frac{1}{\mu\left(\frac{3}{2} Q\right)} \int_{Q}|I(z)| \mathrm{d} \mu(z) \\
& \leq \prod_{i=1}^{m}\left(\frac{1}{\mu\left(\frac{3}{2} Q\right)} \int_{Q}\left|b_{i}(z)-m_{\tilde{Q}}\left(b_{i}\right)\right|^{\tau_{i}} \mathrm{~d} \mu(z)\right)^{\frac{1}{\tau_{i}}}\left(\frac{1}{\mu\left(\frac{3}{2} Q\right)} \int_{Q}\left|T_{m}(\vec{f})\right|^{\tau} \mathrm{d} \mu(z)\right)^{\frac{1}{\tau}} \\
& \leq C\left\|b_{1}\right\|_{*}\left\|b_{2}\right\|_{*} \cdots\left\|b_{m}\right\|_{*} M_{\tau, \frac{3}{2}}\left(T_{m}(\vec{f})\right)(x) \\
& \text { where we have choose } \tau_{i}>1 \text { such that } \frac{1}{\tau_{1}}+\frac{1}{\tau_{2}}+\cdots+\frac{1}{\tau_{m}}+\frac{1}{\tau}=1 .
\end{aligned}
$$

Similarly, for $\tau>1$, by the Hölder inequality, we also deduce that

$$
\begin{aligned}
& \frac{1}{\mu\left(\frac{3}{2} Q\right)} \int_{Q}|I I(z)| \mathrm{d} \mu(z) \\
& \leq C \sum_{i=1}^{m-1} \sum_{\sigma \in C_{i}^{m}} \prod_{\sigma_{j} \in \sigma}\left\|b_{\sigma_{j}}\right\|_{*} M_{\tau, \frac{3}{2}}\left(\left(T_{m}\right)_{b_{\sigma^{\prime}}}(\vec{f})\right)(x) .
\end{aligned}
$$

To estimate the integral related to the function $\operatorname{III}(z)$, we split $f_{i}$ as $f_{i}=f_{i}^{0}+f_{i}^{\infty}$, where $f_{i}^{0}=f \chi_{\frac{4}{3} Q}$ and $f_{i}^{\infty}=f_{i}-f_{i}^{0}$, this yields

$$
\begin{aligned}
\prod_{j=1}^{m} f_{j}\left(y_{j}\right) & =\sum_{\alpha_{1}, \alpha_{2}, \cdots, \alpha_{m} \in\{0, \infty\}} f_{1}^{\alpha_{1}}\left(y_{1}\right) \cdots f_{m}^{\alpha_{m}}\left(y_{m}\right) \\
& =\prod_{j=1}^{m} f_{j}^{0}\left(y_{j}\right)+\sum_{*} f_{1}^{\alpha_{1}}\left(y_{1}\right) \cdots f_{m}^{\alpha_{m}}\left(y_{m}\right)+\prod_{j=1}^{m} f_{j}^{\infty}\left(y_{j}\right),
\end{aligned}
$$

where each term in $\sum$ satisfies that $\alpha_{j_{1}}=\alpha_{j_{2}}=\cdots=\alpha_{j_{\lambda}}=0$, for some $1 \leq \lambda<m$ and some ${ }^{*}\left\{j_{1}, j_{2}, \cdots, j_{\lambda}\right\} \subset\{1,2, \cdots, m\}$. So we can decompose the function $I I I(z)$ further into three parts as follows

$$
\begin{aligned}
I I I(z) \leq & \left|T_{m}\left(\left(b_{1}-m_{\tilde{Q}}\left(b_{1}\right)\right) f_{1}^{0}, \cdots,\left(b_{m}-m_{\tilde{Q}}\left(b_{m}\right)\right) f_{m}^{0}\right)(z)\right| \\
& +\sum_{*}\left|T_{m}\left(\left(b_{1}-m_{\tilde{Q}}\left(b_{1}\right)\right) f_{1}^{\alpha_{1}}, \cdots,\left(b_{m}-m_{\tilde{Q}}\left(b_{m}\right)\right) f_{1}^{\alpha_{m}}\right)(z)\right| \\
& +\left|(-1)^{m} T_{m}\left(\left(b_{1}-m_{\tilde{Q}}\left(b_{1}\right)\right) f_{1}^{\infty}, \cdots,\left(b_{m}-m_{\tilde{Q}}\left(b_{m}\right)\right) f_{1}^{\infty}\right)(z)-h_{Q}\right| \\
= & : I I I_{1}(z)+I I I_{2}(z)+I I I_{3}(z) .
\end{aligned}
$$

For $s_{i}>1$,we can take $1<\mu_{i}<s_{i}$ such that $\frac{1}{v}=\frac{1}{u_{1}}+\frac{1}{u_{2}}+\cdots+\frac{1}{u_{m}}$ and $v>1$. Let $\frac{1}{u_{i}}=\frac{1}{s_{i}}+\frac{1}{t_{i}}$ for each $i=1,2, \cdots, m$, then $1<t_{i}<\infty$. Using Lemma 2.4, we know that the $T_{m}$ is bounded from $L^{u_{1}}(\mu) \times L^{u_{2}}(\mu) \times \cdots \times L^{u_{m}}(\mu)$ to $L^{v}(\mu)$. Hence, by this boundedness and Hölder inequality, we have 


$$
\begin{aligned}
& \frac{1}{\mu\left(\frac{3}{2} Q\right)} \int_{Q}\left|I I I_{1}(z)\right| \mathrm{d} \mu(z) \\
& \leq \frac{\mu(Q)^{1-\frac{1}{v}}}{\mu\left(\frac{3}{2} Q\right)}\left\|T_{m}\left(\left(b_{1}-m_{\tilde{Q}}\left(b_{1}\right)\right) f_{1}^{0}, \cdots,\left(b_{m}-m_{\tilde{Q}}\left(b_{m}\right)\right) f_{m}^{0}\right)(z)\right\|_{L^{v}(\mu)} \\
& \leq C \mu\left(\frac{3}{2} Q\right)^{-\frac{1}{v}} \prod_{i=1}^{m}\left\|\left(b_{i}-m_{\tilde{Q}}\left(b_{i}\right)\right) f_{i}^{0}\right\|_{L^{u_{i}}(\mu)} \\
& \leq C \prod_{i=1}^{m}\left\|b_{i}\right\|_{*} M_{s_{i}, \frac{9}{8}} f_{i}(x) .
\end{aligned}
$$

In order to estimate the integral of terms $\mathrm{III}_{2}(z)$ and $\mathrm{III}_{3}(z)$ over $Q$, we will give their point-wise estimates. In fact, for $z \in Q$, since $1 \leq \lambda \leq m-1$ we observe that

$$
\begin{aligned}
I I I_{2}(z)= & \sum_{*}\left|T_{m}\left(\left(b_{1}-m_{\tilde{Q}}\left(b_{1}\right)\right) f_{1}^{\alpha_{1}}, \cdots,\left(b_{m}-m_{\tilde{Q}}\left(b_{m}\right)\right) f_{1}^{\alpha_{m}}\right)(z)\right| \\
\leq & C \sum_{*} \prod_{j \in\left\{j_{1}, \cdots, j_{\lambda}\right\}} \int_{\frac{4}{3} Q}\left|b_{j}\left(y_{j}\right)-m_{\tilde{Q}}\left(b_{j}\right)\right|\left|f_{j}\left(y_{j}\right)\right| \mathrm{d} \mu\left(y_{j}\right) \\
& \left.\times \int_{\left(\mathbb{R}^{d} \backslash \frac{4}{3} Q\right.} Q\right)^{m-\lambda} \frac{\sum_{j \notin\left\{j_{1}, \cdots, j_{\lambda}\right\}}\left|b_{j}\left(y_{j}\right)-m_{\tilde{Q}}\left(b_{j}\right)\right|\left|f_{j}\left(y_{j}\right)\right| \mathrm{d} \mu\left(y_{j}\right)}{\left(\sum_{j \notin\left\{j_{1}, \cdots, j_{\lambda}\right\}}\left|z-y_{j}\right|\right)^{m n}} \\
\leq & C \sum_{*} \prod_{j \in\left\{j_{1}, j_{2}, \cdots, j_{\lambda}\right\}}\left\|b_{j}\right\|_{*} M_{s_{j}, \frac{9}{8}} f_{j}(x) \\
& \times \sum_{k=1}^{\infty} 2^{-k \lambda n} \prod_{j \notin\left\{j_{1}, j_{2}, \cdots, j_{\lambda}\right\}}\left\|b_{j}\right\|_{*}(k+1) M_{s_{j}, \frac{9}{8}} f_{j}(x) \\
\leq & C \prod_{j=1}^{\infty}\left\|b_{j}\right\|_{*} M_{s_{j}, \frac{9}{8}} f_{j}(x)
\end{aligned}
$$

where we have used the fact (see[5]) that, there is an absolute constant $C$ such that, for any $b \in R B M O$, integer $k \geq 0$ and cubes $Q$,

$$
\begin{aligned}
\left|m_{2^{2^{\frac{4}{3}}} \frac{1}{3}}(b)-m_{\tilde{Q}}(b)\right| & \leq C\|b\|_{*} K_{\tilde{Q}, 2^{k^{\frac{4}{3}} Q}} \\
& \leq C\|b\|_{*} K_{Q, 2^{\frac{4}{3}} \frac{4}{3} Q} \\
& \leq C k\|b\|_{*} .
\end{aligned}
$$

On the other hand, for $\mathrm{III}_{3}(z)$, we note for any $z, y \in Q$ that

$$
\begin{aligned}
& \mid T_{m}\left(\left(b_{1}-m_{\tilde{Q}}\left(b_{1}\right)\right) f_{1}^{\infty}, \cdots,\left(b_{m}-m_{\tilde{Q}}\left(b_{m}\right)\right) f_{m}^{\infty}\right)(z) \\
& -T_{m}\left(\left(b_{1}-m_{\tilde{Q}}\left(b_{1}\right)\right) f_{1}^{\infty}, \cdots,\left(b_{m}-m_{\tilde{Q}}\left(b_{m}\right)\right) f_{m}^{\infty}\right)(y) \mid \\
& \leq \int_{\left(\mathbb{R}^{d}\left(\frac{4}{3} Q\right)^{m}\right.}\left|K\left(z, y_{1}, \cdots, y_{m}\right)-K\left(y, y_{1}, \cdots, y_{m}\right)\right| \\
& \quad \times\left|\prod_{i=1}^{m}\left(b_{i}\left(y_{i}\right)-m_{\tilde{Q}}\left(b_{i}\right)\right) f_{i}^{\infty}\right| \mathrm{d} \mu\left(y_{1}\right) \mathrm{d} \mu\left(y_{2}\right) \cdots \mathrm{d} \mu\left(y_{m}\right)
\end{aligned}
$$




$$
\begin{aligned}
& \left.\leq \int_{\left(\mathbb{R}^{d} \backslash \frac{4}{3} Q\right.} Q\right)^{m} \frac{|z-y|^{\epsilon} \prod_{i=1}^{m}\left|\left(b_{i}\left(y_{i}\right)-m_{\tilde{Q}}\left(b_{i}\right)\right) f_{i}^{\infty}\right|}{\left(\sum_{k \cdot l=0}^{m}\left|y_{k}-y_{l}\right|\right)^{m n+\epsilon}} \mathrm{d} \mu\left(y_{1}\right) \cdots \mathrm{d} \mu\left(y_{m}\right) \\
& \leq C \prod_{i=1}^{m} \sum_{k=1}^{\infty} \int_{2^{k} \frac{4}{3} Q \backslash 2^{k-1} \frac{4}{3} Q} \frac{l(Q)^{\frac{\epsilon}{m}}}{l\left(2^{k} \frac{3}{2} Q\right)^{n+\frac{\epsilon}{m}}}\left|\left(b_{i}\left(y_{i}\right)-m_{\tilde{Q}}\left(b_{i}\right)\right) f_{i}\right| \mathrm{d} \mu\left(y_{i}\right) \\
& \leq C \prod_{i=1}^{m} \sum_{k=1}^{\infty} 2^{-\frac{k}{m}}(k+1)\left\|b_{i}\right\|_{*} M_{s_{i}, \frac{9}{8}} f_{i}(x) \\
& \leq C \prod_{i=1}^{m}\left\|b_{i}\right\|_{*} M_{s_{i}, \frac{9}{8}} f_{i}(x),
\end{aligned}
$$

where we have use the inequation (33) again.

Taking the mean over $y \in Q$, we can obtain that

$$
\frac{1}{\mu\left(\frac{3}{2} Q\right)} \int_{Q}\left(\left|I I I_{2}(z)\right|+\left|I I I_{3}(z)\right|\right) \mathrm{d} \mu(z) \leq C \prod_{i=1}^{m}\left\|b_{i}\right\|_{*} M_{s_{i}, \frac{9}{8}} f_{i}(x) .
$$

Combing the inequalities (26) (27), (28), (30), (31) and (33), we see from the estimates of $I, I I, I I I_{1}, I I I_{2}$ and $I I I_{3}$ that the desired inequality (22) holds.

Next we turn to estimate the inequality (23). For any cubes $Q \subset R$ with $x \in Q$, where $R$ is doubling. We denote $N_{Q, R}+2$ by $N$, then $2^{N} Q \supset 2 Q$ and $2^{N} Q \supset 2 R$. We recall the equality (29) and let $f_{i}^{0}=f_{i} \chi_{2^{N} Q \backslash \frac{4}{3} Q}$ and $f_{i}^{R}=f_{i} \chi_{2^{N} Q \frac{4}{3} R}$, and let $f_{i}^{\infty}=f_{i} \chi_{\mathbb{R}^{d} 12^{N} Q}$. Then we can write

$$
\begin{aligned}
&\left|h_{Q}-h_{R}\right|=\mid m_{Q}\left[T_{m}\left(\left(m_{\tilde{Q}}\left(b_{1}\right)-b_{1}\right) f_{1} \chi_{\mathbb{R}^{d}, \frac{4}{3} Q}, \cdots,\left(m_{\tilde{Q}}\left(b_{m}\right)-b_{m}\right) f_{m} \chi_{\mathbb{R}^{d}, \frac{4}{3} Q}\right)\right] \\
&-m_{R}\left[T_{m}\left(\left(m_{\tilde{R}}\left(b_{1}\right)-b_{1}\right) f_{1} \chi_{\mathbb{R}^{d}, \frac{4}{3} R}, \cdots,\left(m_{\tilde{R}}\left(b_{m}\right)-b_{m}\right) f_{m} \chi_{\mathbb{R}^{d}, \frac{4}{3} R}\right)\right] \mid \\
& \leq \mid m_{Q}\left[T_{m}\left(\left(m_{\tilde{Q}}\left(b_{1}\right)-b_{1}\right) f_{1}^{\infty}, \cdots,\left(m_{\tilde{Q}}\left(b_{m}\right)-b_{m}\right) f_{m}^{\infty}\right)\right] \\
&-m_{R}\left[T_{m}\left(\left(m_{\tilde{Q}}\left(b_{1}\right)-b_{1}\right) f_{1}^{\infty}, \cdots,\left(m_{\tilde{Q}}\left(b_{m}\right)-b_{m}\right) f_{m}^{\infty}\right)\right] \mid \\
&+\mid m_{R}\left[T_{m}\left(\left(m_{\tilde{Q}}\left(b_{1}\right)-b_{1}\right) f_{1}^{\infty}, \cdots,\left(m_{\tilde{Q}}\left(b_{m}\right)-b_{m}\right) f_{m}^{\infty}\right)\right] \\
&-m_{R}\left[T_{m}\left(\left(m_{R}\left(b_{1}\right)-b_{1}\right) f_{1}^{\infty}, \cdots,\left(m_{R}\left(b_{m}\right)-b_{m}\right) f_{m}^{\infty}\right)\right] \mid \\
&+\sum_{\substack{\alpha_{1}, \alpha_{2}, \cdots, \alpha_{m} \in\{0, \infty) \\
\text { at least one } \alpha_{i} \neq \infty}}\left|m_{Q}\left[T_{m}\left(\left(m_{\tilde{Q}}\left(b_{1}\right)-b_{1}\right) f_{1}^{\alpha_{1}}, \cdots,\left(m_{\tilde{Q}}\left(b_{m}\right)-b_{m}\right) f_{m}^{\alpha_{m}}\right)\right]\right| \\
&+\sum_{\substack{\alpha_{1}, \alpha_{2}, \cdots, \alpha_{m} \in\left\{R, \infty \infty \\
\text { at least one } \alpha_{i} \neq \infty\right.}}\left|m_{R}\left[T_{m}\left(\left(m_{R}\left(b_{1}\right)-b_{1}\right) f_{1}^{\alpha_{1}}, \cdots,\left(m_{R}\left(b_{m}\right)-b_{m}\right) f_{m}^{\alpha_{m}}\right)\right]\right| \\
&= A_{1}+A_{2}+A_{3}+A_{4} .
\end{aligned}
$$

For the term $A_{1}$, noting 


$$
\left|m_{R}\left(b_{i}\right)-m_{\tilde{Q}}\left(b_{i}\right)\right| \leq C\left\|b_{i}\right\|_{*} K_{Q, R}
$$

and the similar argument as that for the estimate of $\mathrm{III}_{3}$, we can obtain that

$$
A_{1} \leq C \prod_{i=1}^{m} K_{Q, R}\left\|b_{i}\right\|_{*} M_{s_{i}, \frac{9}{8}} f_{i}(x) \leq C\left(K_{Q, R}\right)^{m} \prod_{i=1}^{m}\left\|b_{i}\right\|_{*} M_{s_{i}, \frac{9}{8}} f_{i}(x) .
$$

To estimate $A_{2}$, we recall the notations and note that, for any sequences $\xi_{j}$ and $\zeta_{j}$,

$$
\prod_{j=1}^{m}\left(\xi_{j}+\zeta_{j}\right)=\sum_{i=0}^{m} \sum_{\sigma \in C_{i}^{m}} \prod_{j \in \sigma} \xi_{j} \prod_{j^{\prime} \in \sigma^{\prime}} \zeta_{j^{\prime}}
$$

Using this equality and expanding

$$
\begin{aligned}
m_{\tilde{Q}}\left(b_{j}\right)-b_{j}(y) & =\left[m_{\tilde{Q}}\left(b_{j}\right)-b_{j}(z)\right]+\left[b_{j}(z)-b_{j}(y)\right] \text {, we observe that } \\
& T_{m}\left(\left(m_{\tilde{Q}}\left(b_{1}\right)-b_{1}\right) f_{1}^{\infty}, \cdots,\left(m_{\tilde{Q}}\left(b_{m}\right)-b_{m}\right) f_{m}^{\infty}\right)(z) \\
= & \sum_{i=0}^{m} \sum_{\sigma \in C_{i}^{m}} \prod_{j \in \sigma}\left(m_{\tilde{Q}}\left(b_{j}\right)-b_{j}(z)\right)\left(T_{m}\right)_{\prod_{\sigma_{\sigma^{\prime}}}}\left(\vec{f} \chi_{\mathbb{R}^{d} \backslash 2^{N} \mathcal{Q}}\right)(z)
\end{aligned}
$$

Similarly,

$$
\begin{aligned}
& T_{m}\left(\left(m_{R}\left(b_{1}\right)-b_{1}\right) f_{1}^{\infty}, \cdots,\left(m_{R}\left(b_{m}\right)-b_{m}\right) f_{m}^{\infty}\right)(z) \\
& =\sum_{i=0}^{m} \sum_{\sigma \in C_{i}^{m}} \prod_{j \in \sigma}\left(m_{R}\left(b_{j}\right)-b_{j}(z)\right)\left(T_{m}\right)_{\prod \vec{b}_{\sigma^{\prime}}}\left(\vec{f} \chi_{\mathbb{R}^{d} \backslash 2^{N} Q}\right)(z) .
\end{aligned}
$$

Thus

$$
\begin{aligned}
& \mid T_{m}\left(\left(m_{\tilde{Q}}\left(b_{1}\right)-b_{1}\right) f_{1}^{\infty}, \cdots,\left(m_{\tilde{Q}}\left(b_{m}\right)-b_{m}\right) f_{m}^{\infty}\right)(z) \\
& -T_{m}\left(\left(m_{R}\left(b_{1}\right)-b_{1}\right) f_{1}^{\infty}, \cdots,\left(m_{R}\left(b_{m}\right)-b_{m}\right) f_{m}^{\infty}\right)(z) \mid \\
& \leq\left|\sum_{i=1}^{m} \sum_{\sigma \in C_{i}^{m}} \prod_{j \in \sigma}\left(m_{\tilde{Q}}\left(b_{j}\right)-b_{j}(z)\right)\left(T_{m}\right)_{\prod_{b_{\sigma^{\prime}}}}\left(\vec{f} \chi_{\mathbb{R}^{d} 2^{N} Q}\right)(z)\right| \\
& \quad+\left|\sum_{i=1}^{m} \sum_{\sigma \in C_{i}^{m}} \prod_{j \in \sigma}\left(m_{R}\left(b_{j}\right)-b_{j}(z)\right)\left(T_{m}\right)_{\prod_{\sigma_{\sigma^{\prime}}}}\left(\vec{f} \chi_{\mathbb{R}^{d} 2^{N} Q}\right)(z)\right| \\
& =: B_{1}(z)+B_{2}(z) .
\end{aligned}
$$

To estimate the integrals above, we recall that $f_{i}^{\infty}=f_{i} \chi_{\mathbb{R}^{d} \backslash 2^{N} Q}$ and let $f_{j}^{Q}=f_{j} \chi_{2^{N} Q}$, then we can write that $f_{j}^{\infty}=f_{j}-f_{j}^{Q}$ and $f_{j}=f_{j}^{\infty}+f_{j}^{Q}$, and thus we have

$$
\begin{aligned}
& \prod_{j=1}^{m} f_{j}^{\infty}\left(y_{j}\right)=\prod_{j=1}^{m} f_{j}\left(y_{j}\right)+\sum_{i=1}^{m}(-1)^{i} \sum_{\rho \in C_{i}^{m}} \prod_{j \in \rho} f_{j}^{Q}\left(y_{j}\right) \prod_{j^{\prime} \in \rho^{\prime}} f_{j^{\prime}}\left(y_{j^{\prime}}\right) \\
& =\prod_{j=1}^{m} f_{j}\left(y_{j}\right)+\sum_{\lambda=1}^{m} \sum_{\left\{j_{1}, j_{2}, \cdots, j_{\lambda}\right\} \in\{1,2, \cdots, m\}} \\
& \quad \times C_{j_{1}, j_{2}, \cdots, j_{m}} f_{j_{1}}^{Q}\left(y_{j_{1}}\right), \cdots, f_{j_{\lambda}}^{Q}\left(y_{j_{\lambda}}\right) f_{j_{\lambda+1}}^{\infty}\left(y_{j_{\lambda+1}}\right), \cdots, f_{j_{m}}^{\infty}\left(y_{j_{m}}\right)
\end{aligned}
$$

where $C_{j_{1}, j_{2}, \cdots, j_{m}}$ are constant independent of $\vec{f}$ and Q. From the equality (43), we can deduce that 


$$
\begin{aligned}
& \left(T_{m}\right)_{\prod_{\sigma^{\prime}}}\left(\vec{f} \chi_{\mathbb{R}^{d} \backslash 2^{N} Q}\right)(z) \\
& =\left(T_{m}\right)_{\prod \vec{b}_{\sigma^{\prime}}} \vec{f}(z)+\sum_{\lambda=1}^{m} \sum_{\left\{j_{1}, j_{2}, \cdots, j_{m}\right\}<\{1,2, \cdots, m\}} C_{j_{1}, j_{2}, \cdots, j_{m}} T_{m}\left(g_{1} F_{1}, g_{2} F_{2}, \cdots, g_{m} F_{m}\right)(z)
\end{aligned}
$$

where

$$
g_{j}(y)= \begin{cases}b_{j}(z)-b_{j}(y), & \text { if } j \in \sigma^{\prime} \\ 1, & \text { if } j \in \sigma\end{cases}
$$

and

$$
F_{j}(y)= \begin{cases}f_{j}^{Q}(y), & \text { if } j \in\left\{j_{1}, \cdots, j_{\lambda}\right\} \\ f_{j}^{\infty}(y), & \text { if } j \notin\left\{j_{1}, \cdots, j_{\lambda}\right\}\end{cases}
$$

Along the same lines as that of the pointwise estimates of $I I I_{2}(z)$,we can obtain that, for $x, z \in R \subset 2^{N-1} Q$ and if $1 \leq \lambda \leq m$,

$$
\begin{aligned}
& \left|T_{m}\left(g_{1} F_{1}, g_{2} F_{2}, \cdots, g_{m} F_{m}\right)(z)\right| \\
& \leq C \prod_{j \in\left\{j_{1}, j_{2}, \cdots, j_{\lambda}\right\}} \frac{1}{l\left(2^{N} Q\right)^{n}} \int_{2^{N} Q}\left|g_{j}\left(y_{j}\right) f_{j}\left(y_{j}\right)\right| \mathrm{d} \mu\left(y_{j}\right) \\
& \times \sum_{k=1}^{\infty} 2^{-k \lambda n} \prod_{\substack{j \notin\left\{j_{1}, j_{2}, \cdots, j_{\lambda}\right\} \\
1 \leq j \leq m}} \frac{1}{l\left(2^{k} 2^{N} Q\right)^{n}} \int_{2^{k_{2} N} Q}\left|g_{j}\left(y_{j}\right) f_{j}\left(y_{j}\right)\right| \mathrm{d} \mu\left(y_{j}\right) .
\end{aligned}
$$

Let $\left(g_{j}\right)_{k, s_{j}^{\prime}}^{*}=1$ if $j \in \sigma$; and

$$
\left(g_{j}\right)_{k, s_{j}^{\prime}}^{*}= \begin{cases}\left(\frac{1}{l\left(2^{N} Q\right)^{n}} \int_{2^{N} Q}\left|b_{j}(z)-b_{j}(y)\right|^{s_{j}^{\prime}} \mathrm{d} \mu(y)\right)^{\frac{1}{s_{j}^{\prime}}}, & \text { if } j \in \sigma^{\prime} \cap\left\{j_{1}, j_{2}, \cdots, j_{\lambda}\right\} \\ \left(\frac{1}{l\left(2^{k+N} Q\right)^{n}} \int_{2^{k+N} Q}\left|b_{j}(z)-b_{j}(y)\right|^{s_{j}^{\prime}} \mathrm{d} \mu(y)\right)^{\frac{1}{s_{j}^{\prime}}}, & \text { if } j \in \sigma^{\prime} \backslash\left\{j_{1}, j_{2}, \cdots, j_{\lambda}\right\}\end{cases}
$$

thus for $j \in \sigma^{\prime}$,

$$
\left(g_{j}\right)_{k, s_{j}^{\prime}}^{*} \leq C\left\|b_{j}\right\|_{*}+C\left|m_{2^{N} Q}\left(b_{j}\right)-b_{j}(z)\right|+C\left|m_{2^{k+N}} Q\left(b_{j}\right)-b_{j}(z)\right| .
$$

Hence we get from (45) that, for $\tau>1$,

$$
\begin{aligned}
& \left(\frac{1}{\mu(R)} \int_{R}\left|T_{m}\left(g_{1} F_{1}, g_{2} F_{2}, \cdots, g_{m} F_{m}\right)(z)\right|^{\tau} \mathrm{d} \mu(z)\right)^{\frac{1}{\tau}} \\
& \leq C\left(\prod_{j=1}^{m} M_{s_{j}, \frac{9}{8}} f_{j}(x)\right) \sum_{j=1}^{\infty} 2^{-k \lambda n} \times\left(\frac{1}{\mu(R)} \int_{R}\left|\prod_{j \in \sigma^{\prime}}\left(g_{j}\right)_{k, s_{j}^{\prime}}^{*}\right|^{\tau} \mathrm{d} \mu(z)\right)^{\frac{1}{\tau}} \\
& \leq C\left(\prod_{j=1}^{m} M_{s_{j}, \frac{9}{8}} f_{j}(x)\right) \sum_{j=1}^{\infty} 2^{-k \lambda n} \prod_{j \in \sigma^{\prime}}\left\|b_{j}\right\|_{*}\left(1+K_{R, 2^{N} Q}+K_{R, 2^{k+N} Q}\right) \\
& \leq C\left(\prod_{j=1}^{m} M_{s_{j}, \frac{9}{8}} f_{j}(x)\right) \prod_{j \in \sigma^{\prime}}\left\|b_{j}\right\|_{*},
\end{aligned}
$$

where we have used the fact that the cubes $R$ and $2^{N} Q$ are comparable, which 
implies $K_{R, 2^{N} Q} \leq C$ and $K_{R, 2^{k+N} Q} \leq C(1+k)$. Using the inequality (46) above and the identity (44), we obtain that, for $\tau>1$,

$$
\begin{aligned}
A_{2} \leq & \frac{1}{\mu(R)} \int_{R}\left(\left|B_{1}(z)\right|+\left|B_{2}(z)\right|\right) \mathrm{d} \mu(z) \\
\leq & C \sum_{i=1}^{m} \sum_{\sigma \in C_{i}^{m}}\left(\prod_{j \in \sigma} K_{Q, R}\left\|b_{j}\right\|_{*}\right) \\
& \times\left(\frac{1}{\mu(R)} \int_{R}\left|\left(T_{m}\right)_{\prod_{\sigma_{\sigma^{\prime}}}}\left(\vec{f} \chi_{\mathbb{R}^{d} \underline{2}^{N} Q}\right)\right|^{\tau} \mathrm{d} \mu(z)\right)^{\frac{1}{\tau}} \\
\leq & C \sum_{i=1}^{m} \sum_{\sigma \in C_{i}^{m}}\left(\prod_{j \in \sigma} K_{Q, R}\left\|b_{j}\right\|_{*}\right) M_{\tau, \frac{3}{2}}\left(\left(T_{m}\right)_{\prod \vec{b}_{\sigma^{\prime}}}(\vec{f})\right)(x) \\
& +C \prod_{j=1}^{m} K_{Q, R}\left\|b_{j}\right\|_{*} M_{s_{j}, \frac{9}{8}} f_{j}(x) .
\end{aligned}
$$

The estimates of $A_{3}$ and $A_{4}$ is very similar to the one used in the estimate of $A_{2}$. In fact, repeating the similar procedures used in (45) and (46) for $\tau>1$, and noting that $K_{Q, 2^{k+N} Q} \leq K_{Q, R}+K_{R, 2^{k+N} Q} \leq C(1+k)+K_{Q, R}$ since $2^{N-3} Q \subset 2 R \subset 2^{N} Q$ by the definition of $N$, we can deduce that

$$
\begin{aligned}
& \sum_{\substack{\left.\alpha_{1}, \alpha_{2}, \cdots, \alpha_{m} \in\{0, \infty)\right\} \\
\text { at least on } \alpha_{i} \neq \infty \\
\text { and one } \alpha_{j} \neq 0}}\left|m_{Q}\left[T_{m}\left(\left(m_{\tilde{Q}}\left(b_{1}\right)-b_{1}\right) f_{1}^{\alpha_{1}}, \cdots,\left(m_{\tilde{Q}}\left(b_{m}\right)-b_{m}\right) f_{m}^{\alpha_{m}}\right)\right]\right| \\
& +\sum_{\substack{\alpha_{1}, \alpha_{2}, \cdots, \alpha_{m} \in\left\{R, \infty, \infty \\
\text { at least one } \alpha_{i} \neq \infty\right.}}\left|m_{R}\left[T_{m}\left(\left(m_{R}\left(b_{1}\right)-b_{1}\right) f_{1}^{\alpha_{1}}, \cdots,\left(m_{R}\left(b_{m}\right)-b_{m}\right) f_{m}^{\alpha_{m}}\right)\right]\right| \\
& \leq C \prod_{j=1}^{\alpha_{1}} K_{Q, R}\left\|b_{j}\right\|_{*} M_{s_{j}, \frac{9}{8}} f_{j}(x) .
\end{aligned}
$$

It is left to estimate the term in $A_{3}$ of the case $\alpha_{1}=\alpha_{2}=\cdots=\alpha_{m}=0$. A small modification is needed to estimate this term. For $z \in Q$ and $x \in Q$, one sees

$$
\begin{aligned}
& \left|T_{m}\left(\left(m_{\tilde{Q}}\left(b_{1}\right)-b_{1}\right) f_{1}^{0}, \cdots,\left(m_{\tilde{Q}}\left(b_{j}\right)-b_{j}\right) f_{j}^{0}, \cdots,\left(m_{\tilde{Q}}\left(b_{m}\right)-b_{m}\right) f_{m}^{0}\right)(z)\right| \\
& \leq C \sum_{k=1}^{N} \prod_{j=1}^{m} \frac{1}{l\left(2^{k} \frac{4}{3} Q\right)^{n}} \int_{2^{k} \frac{4}{3} Q}\left|\left(m_{\tilde{Q}}\left(b_{j}\right)-b_{j}\left(y_{j}\right)\right) f_{j}\left(y_{j}\right)\right| \mathrm{d} \mu\left(y_{j}\right) \\
& \leq C \sum_{k=1}^{N} \prod_{j=1}^{m} l\left(2^{k} \frac{4}{3} Q\right)^{n} \\
& \leq C\left(K_{Q, R}\right)^{2 m} \prod_{j=1}^{m}\left\|b_{j}\right\|_{*} M_{s_{j,}, \frac{9}{8}} f_{j}(x) .
\end{aligned}
$$

This and the inequality (47) follows

$$
A_{3}+A_{4} \leq C\left(K_{Q, R}\right)^{2 m} \prod_{j=1}^{m}\left\|b_{j}\right\|_{*} M_{s_{j}, \frac{9}{8}} f_{j}(x) .
$$

Moreover, combing the estimates of $A_{1}, A_{2}, A_{3}$ and $A_{4}$, we obtain the de- 
sired inequality (23).

Finally, let us show how to acquire the inequality (19) from the two inequalities (22) and (23). Fix the point $x$ and let $Q$ be any cube that $x \in Q$. notice $K_{Q, \tilde{Q}} \leq C$, hence we see from the inequalities (22) and (23) that

$$
\begin{aligned}
& \frac{1}{\mu\left(\frac{3}{2} Q\right)} \int_{Q}\left|\left(T_{m}\right)_{\Pi \vec{b}}(\vec{f})(z)-m_{\tilde{Q}}\left(\left(T_{m}\right)_{\Pi \vec{b}}(\vec{f})\right)\right| \mathrm{d} \mu(z) \\
& \leq \frac{1}{\mu\left(\frac{3}{2} Q\right)} \int_{Q}\left|\left(T_{m}\right)_{\Pi \vec{b}}(\vec{f})(z)-h_{Q}\right| \mathrm{d} \mu(z)+\frac{1}{\mu\left(\frac{3}{2} Q\right)} \int_{Q}\left|h_{Q}-h_{\tilde{Q}}\right| \mathrm{d} \mu(z) \\
& +\frac{1}{\mu\left(\frac{3}{2} Q\right)} \int_{Q}\left|m_{\tilde{Q}}\left(\left(T_{m}\right)_{\Pi \vec{b}}(\vec{f})\right)-h_{\tilde{Q}}\right| \mathrm{d} \mu(z) \\
& \leq C \mathcal{R}(\vec{b}, \vec{f})(x) .
\end{aligned}
$$

On the other hand for all doubling cubes $Q \subset R$ with $x \in Q$ such that $K_{Q, R} \leq P_{0}$, where $P_{0}$ is the constant in Lemma 6 in [15], using (23), we have

$$
\left|h_{Q}-h_{R}\right| \leq C K_{Q, R} P_{0}^{2 m-1} \mathcal{R}(\vec{b}, \vec{f})(x)
$$

and moreover the inequality (49) holds for any doubling cubes $Q, R$ with $Q \subset R$. Therefore,

$$
\begin{aligned}
& m_{Q}\left(\left(T_{m}\right)_{\Pi \vec{b}}(\vec{f})\right)-m_{R}\left(\left(T_{m}\right)_{\Pi^{b}}(\vec{f})\right) \mid \\
& \leq\left|m_{Q}\left(\left(T_{m}\right)_{\Pi_{\vec{b}}}(\vec{f})\right)-h_{Q}\right|+\left|h_{R}-m_{R}\left(\left(T_{m}\right)_{\Pi_{\vec{b}}}(\vec{f})\right)\right|+\left|h_{Q}-h_{R}\right| \\
& \leq C K_{Q, R} \mathcal{R}(\vec{b}, \vec{f})(x) .
\end{aligned}
$$

According to the estimates (48) (50) and the definition of the sharp maximal function, we deduce the inequality (19) and so finish the proof of the Lemma 2.5. $\square$

\section{Conclusions}

The proof of Lemma 2.5 can be slightly modified to prove the conclusion of Lemma 2.1. Therefore we show that the iterated commutators generated by multilinear singular integrals operators $\left(T_{m}\right)_{\Pi_{\vec{b}}}$ are bounded from $M_{q_{1}}^{p_{1}}(\mu) \times \cdots \times M_{q_{m}}^{p_{m}}(\mu)$ to $M_{q}^{p}(\mu)$. Suppose $\|\mu\|=\infty$, the detailed conclusion can be described as follows: Let $\left(T_{m}\right)_{\prod_{\vec{b}}}$ as in (9) and satisfying conditions (7) and (8). Let $1<q_{j} \leq p_{j}<\infty$ with $\frac{1}{p}=\frac{1}{p_{1}}+\cdots+\frac{1}{p_{m}}$ and $\frac{1}{q}=\frac{1}{q_{1}}+\cdots+\frac{1}{q_{m}}$. Suppose $b_{i} \in R B M O(\mu)$ for $i=1,2, \cdots, m$. If $T_{m}$ maps $L^{1}(\mu) \times \cdots \times L^{1}(\mu)$ to $L^{1 / m, \infty}(\mu)$, then the commutators $\left(T_{m}\right)_{\Pi^{b} \vec{b}}$ are bounded from $M_{q_{1}}^{p_{1}}(\mu) \times \cdots \times M_{q_{m}}^{p_{m}}(\mu)$ to $M_{q}^{p}(\mu)$, that is,

$$
\left\|\left(T_{m}\right)_{\prod^{\vec{b}}}(\vec{f})(x)\right\|_{M_{q}^{p}(\mu)} \leq C \prod_{i=1}^{m}\left\|b_{i}\right\|_{*}\left\|f_{i}\right\|_{M_{q_{i}}^{p_{i}}(\mu)} .
$$

More generally, let $\left(T_{m}\right)_{\prod_{\vec{b}_{\sigma}}}$ as in (11) and satisfying conditions (7) and (8). 
Let $1<q_{j} \leq p_{j}<\infty$ with $\frac{1}{p}=\frac{1}{p_{1}}+\cdots+\frac{1}{p_{m}}$ and $\frac{1}{q}=\frac{1}{q_{1}}+\cdots+\frac{1}{q_{m}}$. Suppose

$b_{i} \in R B M O(\mu)$ for $i=1,2, \cdots, m$. If $T_{m}$ maps $L^{1}(\mu) \times \cdots \times L^{1}(\mu)$ to

$L^{1 / m, \infty}(\mu)$, then for all $\sigma \subset\{1,2, \cdots, m\}$, the commutators $\left(T_{m}\right)_{\Pi \vec{b}_{\sigma}}$ are bounded from $M_{q_{1}}^{p_{1}}(\mu) \times \cdots \times M_{q_{m}}^{p_{m}}(\mu)$ to $M_{q}^{p}(\mu)$, that is,

$$
\left\|\left(T_{m}\right)_{\prod \vec{b}_{\sigma}}(\vec{f})(x)\right\|_{M_{q}^{p}(\mu)} \leq C \prod_{j \in \sigma}\left\|b_{j}\right\|_{\|_{i}} \prod_{i=1}^{m}\left\|f_{i}\right\|_{M_{q_{i}}^{p_{i}}(\mu)} .
$$

\section{Funding}

Supported by the National Natural Science Foundation of China (Grant No. 11161044 and Grant No. 11661061) and Natural science foundation of Inner Mongolia (No. 2019MS01003).

\section{Conflicts of Interest}

The authors declare no conflicts of interest regarding the publication of this paper.

\section{References}

[1] Nazarov, F., Treil, S. and Volberg, A. (1997) Cauchy Integral and Calderón-Zygmund Operators on Nonhomogeneous Spaces. International Mathematics Research Notices, 15, 703-726. https://doi.org/10.1155/S1073792897000469

[2] Nazarov, F., Treil, S. and Volberg, A. (1998) Weak Type Estimates and Cotlar Inequalities for Calderón-Zygmund Operators on Nonhomogeneous Spaces. International Mathematics Research Notices, 1998, 463-487. https://doi.org/10.1155/S1073792898000312

[3] Tolsa, X. (2003) Painlevés Problem and the Semiadditivity of Analytic Capacity. Acta Mathematica, 190, 105-149. https://doi.org/10.1007/BF02393237

[4] Tolsa, X. (2003) Bilipschitz Maps, Analytic Capacity, and the Cauchy Integral. Annals of Mathematics, 190, 1243-1304. https://doi.org/10.4007/annals.2005.162.1243

[5] Tolsa, X. (2001) BMO, $\mathrm{H}_{1}$, and Calderón-Zygmund Operators for Non Doubling Measures. Mathematische Annalen, 319, 89-149. https://doi.org/10.1007/PL00004432

[6] Tolsa, X. (2003) The Space $\mathrm{H}_{1}$ for Nondoubling Measures in Terms of a Grand Maximal Operator. Transactions of the American Mathematical Society, 355, 315348. https://doi.org/10.1090/S0002-9947-02-03131-8

[7] Tolsa, X. (2001) Littlewood-Paley Theory and the T(1) Theorem with Non-Doubling Measures. Advances in Mathematics, 164, 57-116. https://doi.org/10.1006/aima.2001.2011

[8] Adams, D.R. (1975) A Note on Riesz Potentials. Duke Mathematical Journal, 42, 765-778. https://doi.org/10.1215/S0012-7094-75-04265-9

[9] Chiarenza, F. and Frasca, M. (1987) Morrey Spaces and Hardy-Littlewood Maximal Function. Rendiconti del Seminario Matematico della Università di Padova, 7, 273-279.

[10] Sawano, Y. and Tanaka, H. (2005) Morrey Spaces for Non-Doubling Measures. Acta 
Mathematica Sinica, 21, 1535-1544. https://doi.org/10.1007/s10114-005-0660-Z

[11] Sawano, Y. (2008) Generalized Morrey Spaces for Non-Doubling Measures. Nonlinear Differential Equations and Applications NoDEA, 15, 413-425. https://doi.org/10.1007/s00030-008-6032-5

[12] Coifman, R., Rochberg, R. and Weiss, G. (1976) Factorization Theorems for Hardy Spaces in Several Variables. Annals of Mathematics, 103, 611-635. https://doi.org/10.2307/1970954

[13] Pérez, C. and Trujillo-Gonzalez, R. (2002) Sharp Weighted Estimates for Multilinear Commutators. Journal of the London Mathematical Society, 65, 672-692. https://doi.org/10.1112/S0024610702003174

[14] Hu, G., Meng, Y. and Yang, D. (2005) Multilinear Commutators of Singular Integrals with Non-Doubling Measures. Integral Equations and Operator Theory, 51, 235-255. https://doi.org/10.1007/s00020-003-1251-y

[15] Chen, W. and Sawyer, E. (2002) A Note on Commutators of Fractional Integral with $R B M O(\mu)$ Function. Illinois Journal of Mathematics, 46, 1287-1298. https://doi.org/10.1215/ijm/1258138480

[16] Grafakos, L. and Torres, R. (2002) Multilinear Calderón-Zygmund Theory. Advances in Mathematics, 165, 124-164. https://doi.org/10.1006/aima.2001.2028

[17] Grafakos, L. and Torres, R. (2000) On Multilinear Singular Integrals of CalderońZygmund Type. Proceedings of the 6 th International Conference on Harmonic Analysis and Partial Differential Equations, 57-91. https://doi.org/10.5565/PUBLMAT_Esco02_04

[18] Grafakos, L. and Torres, R. (2002) Maximal Operator and Weighted Norm Inequalities for Multilinear Singular Integrals. Indiana University Mathematics Journal, 51, 1261-1276. https://doi.org/10.1512/iumj.2002.51.2114

[19] Kenig, C. and Stein, E. (1999) Multilinear Estimates and Fractional Integration. Mathematical Research Letters, 6, 1-15. https://doi.org/10.4310/MRL.1999.v6.n1.a1

[20] Pérez, C. and Torres, R. (2003) Sharp Maximal Function Estimates for Multilinear Singular Integrals. In: Harmonic Analysis at Mount Holyoke, Contemporary Mathematics, AMS, Providence, RI, 323-331. https://doi.org/10.1090/conm/320/05615

[21] Pérez, C. and Pradolini, G. (2011) End-Point Estimates for Iterated Commutators of Multilinears of Multilinear Singular Integrals. Classical Analysis and ODEs, arXiv: 1004.4976v2.

[22] Xu, J. (2007) Boundedness in Lebesgue Spaces for Commutators of Multilinear Singular Integrals and RBMO Functions with Non-Doubling Measures. Science in China Series A: Mathematics, 50, 361-376. https://doi.org/10.1007/s11425-007-2073-1

[23] Tao, X. and He, S. (2013) The Boundedness of Multilinear Operators on Generalized Morrey Spaces over the Quasi-Metric Space of Non-Homogeneous Type. Journal of Inequalities and Applications, 2013, Article No. 330. https://doi.org/10.1186/1029-242X-2013-330

[24] Sawano, Y. and Tanaka, H. (2007) Sharp Maximal Inequality and Commutators on Morrey Spaces with Non-Doubling Measures. Taiwanese Journal of Mathematics, 11, 1091-1112. https://doi.org/10.11650/twjm/1500404805

[25] Xu, J. (2007) Boundedness of Multilinear Singular Integrals for Non-Doubling Measures. Journal of Mathematical Analysis and Applications, 327, 471-480. https://doi.org/10.1016/j.jmaa.2006.04.049 